\title{
Ontogenetic changes in the craniomandibular skeleton of the abelisaurid dinosaur Majungasaurus crenatissimus from the Late Cretaceous of Madagascar
}

Nirina O. Ratsimbaholison, Ryan N. Felice, and Patrick M. O'Connor

Acta Palaeontologica Polonica 61 (2), 2016: 281-292 doi:http://dx.doi.org/10.4202/app.00132.2014

Abelisaurid theropods were one of the most diverse groups of predatory dinosaurs in Gondwana during the Cretaceous. The group is characterized by a tall, wide skull and robust cervical region. This morphology is thought to have facilitated specialized feeding behaviors such as prolonged contact with prey. The Late Cretaceous abelisaurid Majungasaurus crenatissimus typifies this abelisaurid cranial morphotype. Recent fossil discoveries of this species include a partial growth series that allows for the first time an investigation of ontogenetic variation in cranial morphology in a representative abelisaurid. Herein we examine growth trajectories in the shape of individual cranial bones and articulated skulls of Majungasaurus using geometric morphometrics. Several major changes in skull shape were observed through ontogeny, including an increase in the height of the jugal, postorbital, and quadratojugal, an increase in the extent of the contacts between bones, and a decrease in the circumference of the orbit. The skull transitions from relatively short in the smallest individual to tall and robust in large adults, as is seen in other theropods. Such morphological change during ontogeny would likely have resulted in different biomechanical properties and feeding behaviors between small and large individuals. These findings provide a post-hatching developmental framework for understanding the evolution of the distinctive tall skull morphology seen in abelisaurids and other large-sized theropod dinosaurs.

Key words: Dinosauria, Abelisauridae, geometric morphometrics, ontogeny, skull, Cretaceous, Gondwana.

Nirina O. Ratsimbaholison [ratsimbano@gmail.com], Department of Paleontology and Biological Anthropology, University of Antananarivo, BP 906, Antananarivo 101, Madagascar; Ohio Center for Ecology and Evolutionary Studies, 228 Irvine Hall, Athens, Ohio 45701 USA. Ryan N. Felice [ryanfelice@ gmail.com ], Ohio Center for Ecology and Evolutionary Studies, 228 Irvine Hall, Athens, Ohio 45701 USA; Department of Biological Sciences, Ohio University, 228 Irvine Hall, Athens, Ohio 45701, USA; current address: Department of Genetics, Evolution and Environment, University College London, London, UK. Patrick M. O'Connor [oconnorp@ ohio.edu] (corresponding author), Ohio Center for Ecology and Evolutionary Studies, 228 Irvine Hall, Athens, Ohio 
45701 USA; Department of Biomedical Sciences, Ohio University Heritage College of Osteopathic Medicine, 228 Irvine Hall, Athens, Ohio 45701, USA.

This is an open-access article distributed under the terms of the Creative Commons

Attribution License (for details please see creativecommons.org), which permits unrestricted use, distribution, and reproduction in any medium, provided the original author and source are credited.

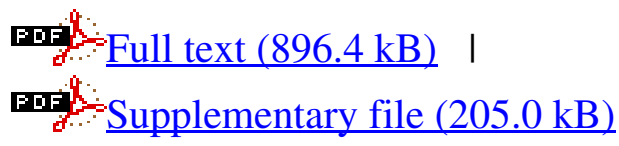

